douleur, nausée et vomissement et du croupe post intubation en salle de réveil. L'anesthésiste doit assumer un rôle actif vis-à-vis des problèmes en salle de réveil et pour congédier les patients externes sinon on devra faire face à une augmentation de l'hospitalisation nonanticipée.

L'anesthésie en externe jouera un rôle de plus en plus important dans les années 90 . Notre but ultime sera d'atteindre le moins d'hospitalisation non prévue possible et de faciliter le retour des patients à leur travail et fonction journalière tout en augmentant leur satisfaction de l'anesthésie.

\section{Day surgical anaesthesia: Which patients? Which procedures?}

Peter G. Duncan MD FRCPC

Department of Anaesthesia, University of Saskatchewan, Saskatoon, Saskatchewan

It has been estimated that between 40 and $50 \%$ of all surgical procedures may be done without overnight postoperative hospitalization. ${ }^{1}$ The safety with which care can be provided in modern outpatient facilities, combined with cost savings inherent in providing ambulatory as opposed to inpatient services, ${ }^{2}$ has resulted in a growing number of procedures being mandated to be done on this basis.

In spite of the widespread development of ambulatory care services there has been relatively little critical evaluation of their outcome. Appropriate randomization of subjects between inpatient and outpatient facilities has not occurred, and many descriptive articles cover a prolonged period during which many changes occurred in both anaesthetic and surgical techniques. Large series of patients from both Canada ${ }^{3,4}$ and the $\mathrm{USA}^{5-7}$ point to the safety of the provision of care on an ambulatory basis. However, the exuberance to reduce hospital costs has resulted in this system of care being invoked for patients beyond those carefully selected in the early development of day surgical programs.

A measure of failure in a day surgical program is the need to retain an unscheduled patient in the hospital. The frequency with which this happens has been reported variously at $1 \%$ in children and $0.28-2.44 \%$ in adults the latter ten-fold variation in complications was most likely related to patient selection and the year of service. The usual reasons for unscheduled admissions relate to persistent pain, nausea and vomiting, syncope, or surgical bleeding. Mortality related to an anaesthetic or surgical problem is distinctly unusual, with emergency or lifethreatening complications estimated to occur in less than $0.007 \%$ of cases. ${ }^{5}$ As to morbidity necessitating admission it has been suggested that surgery is most often at fault: in the large series from Vancouver, admissions occurred for surgical reasons in $0.22 \%$, and for anaesthetic reasons in $0.07 \% .^{3}$ It should not be forgotten that the patient may also be a direct cause, because the lack of an appropriately supportive social environment may necessitate hospitalization and be perceived as failure of the day surgery system.

\section{Which patients?}

The acceptability of patients to a given ambulatory facility will often depend upon the infrastructure in that medical community. The dedication of day surgery units to safety and cost-effectiveness often implies community support resources, the absence of which would preclude ambulatory services. It is impossible, therefore, to generalize completely the recommendations of other units to your own. For example, if responsible home support is lacking, or if transportation distances to obtain postoperative medical assistance are excessive, inpatient care is recommended. Similarly, if the patient is either unable or unwilling to comply with postoperative instructions, acceptance to the day surgical program should be denied.

The descriptive studies referenced have demonstrated several factors that do not have any bearing on outcome. For example, when outcomes are assessed by recovery times or incidence of unscheduled admissions, age does not have a bearing upon outcome (except for expremature babies). Similarly, prolonged anaesthesia does not seem to be a factor, although late recoveries may pose problems for day-surgery staffing. Finally, although most peerreviewed series have included predominately ASA I and II subjects, an increased complication rate with ASA III subjects has not been demonstrated. Indeed, with modern drugs and surgical techniques, patients with wellcontrolled systemic disease are more likely to experience complications related to the surgical procedure than from concurrent medical problems.

There are several types of patients who should not be offered the option of ambulatory care. These include: 1 Non-optimal management of medical disease (ASA III and IV)

2 Malignant hyperthermia 
3 Morbid obesity complicated by haemodynamic or respiratory problems

4 Drug therapy

(a) MAOI

(b) Acute substance abuse

5 Paediatric patients

(a) Expremature infants ( $<45$ weeks)

(b) Lower respiratory tract infections

\section{Which procedures?}

The lists of procedures acceptable for day surgery is constrained only by the need for an uncomplicated postoperative course. In recent years technological advances have revolutionized the number of operations permissible, and will continue to do so. Thus, anecdotal reports of thyroidectomies, vaginal hysterectomies, ectopic pregnancies, and cholecystectomies have been published ${ }^{1}$ as the essential requirements of minimal physiological disturbance, little risk of haemorrhage, and postoperative pain controllable by oral analgesics, are more easily satisfied.

There remain several controversial surgical procedures, such as tonsillectomy in children or angioplasty in adults. While series of successful ambulatory management have been published it is more usual to consider such candidates for "short-stay" (8-24 hr) admissions for observation. Sepsis is also considered a relative complication, for the need for postoperative antibiotic coverage may not be met by non-intravenous techniques.

\section{Conclusion}

What is essential in considering the advisability of ambulatory surgery is a patient-orientated, not procedureorientated, system. Those individuals providing care should be given the option of deciding whether a particular patient is a candidate for any given procedure. If the patient, and those providing the postoperative support, are properly motivated to ensure a course as expected the possibilities are unlimited. However, if the patient's health or home environment leaves any doubt the ambulatory service should not be utilized.

\section{References}

1 Veintraub HD. Patient selection for ambulatory surgery and when do we say no? Advances in Anaesthesia 1989; 6: 47-66.

2 Kitz DS, Slusary-Ladden C, Lecky JH. Hospital resources for inpatient and ambulatory surgery. Anesthesiology 1988; 69: 383-6.

3 Fancourt-Smith PF, Hornstein J, Jenkins LG. Hospital admissions from the day care centre of the Vancouver General Hospital. Can J Anaesth 1990; 37: 699-704.

4 Postuma R, Ferguson CC, Stanwick RS, Horne JM. Ped- iatric day-care surgery: a 30-year hospital experience. J Pediatr Surg: 1987; 22: 304-7.

5 Natof $H E$. Complications associated with ambulatory surgery. JAMA 1980; 244: 1116-8.

6 Meridy $H W$. Criteria for selection of ambulatory surgery patients and guidelines for anesthetic management: a retrospective study of 1553 cases. Anesth Analg 1982; 61: 921-6.

7 Patel RI, Hannallah RS. Anesthetic complications following pediatric ambulatory surgery: a 3-year study. Anesthesiology 1988; 69: 1009-12.

\section{Which is the best anaesthetic technique?}

\author{
Frances Chung MD FRCPC \\ Department of Anaesthesia, The Toronto Hospital, \\ Toronto Western Division, University of Toronto
}

A report of mortality statistics at the 1986 meeting of the Federated Ambulatory Surgery Association revealed 17 deaths in approximately 1.1 million procedures. This emphasizes that although there may be minor surgery, there is no minor anaesthetic. ${ }^{1}$ Recurrent problems occur in two clinical situations, laparoscopy with general anaesthesia, and use of the conscious sedation technique for nasal and facial surgery.

The Federated Ambulatory Surgery Association also had some data on overall complication rates of types of anaesthesia after ambulatory surgery; combined local and sedation anaesthesia techniques (1:106), general (1:120), regional $(1: 277)$ or local anaesthesia alone $(1: 268) .{ }^{2}$ Thus the combined local and sedation group had the highest overall complication rate, and regional or local anaesthesia groups the lowest. It is imperative for the anaesthetist to provide the best anaesthetic care for ambulatory patients to facilitate their return to daily living and work.

\section{How long should we fast our patients?}

In an effort to decrease the incidence of aspiration, patients are routinely asked to be NPO after midnight for surgery scheduled the next day. Recent clinical studies suggest that this overnight fast may not be justified in adults $^{3-5}$ or in children. ${ }^{6-7}$ Pulmonary aspiration usually occurs in emergency abdominal and obstetrical procedures in which there may be such complicating factors as recent food and fluid intake, trauma, administration of narcotic analgesics and difficult intubation. These factors 